\section{Sur une caractérisation des alephs ${ }^{1}$ ).}

\section{Par}

\section{Casimir Kuratowski (Warszawa).}

Étant donné un ensemble $Z$ arbitraire, $Z^{n}$ désigne l'ensemble des suites à $n$ éléments: $z_{1}, z_{2}, \ldots, z_{n}$, où $z_{k} \in Z$ pour $k=1, \ldots, n$. $A$ étant un sous-ensemble de $Z^{n}$, nous dirons que $A$ est de puissance $<\mathrm{m}$ dans la direction $d u$-ème axe, lorsque, quel que soit l'élément $\left(z_{1}, \ldots, z_{k-1}, z_{k+1}, \ldots, z_{n}\right)$ de $Z^{n-1}$, l'ensemble

$$
\underset{z_{k}}{E}\left\{\left(z_{1}, \ldots, z_{k-1}, z_{k}, z_{k+1}, \ldots, z_{n}\right) \in A\right\}
$$

est de puissance <m. (Si $n=1$, cela veut dire, bien entendu, que $\overline{\bar{A}}<\mathrm{m}$ ).

D'après un théorème remarquable de M. Sierpiński ${ }^{2}$ ), l'hypothèse du continu équivaut à l'existence d'une décomposition de l'espace euclidien $\mathcal{E}^{3}$ en trois ensembles $A_{1}, A_{2}, A_{3}$ tels que, pour $k=1,2,3$, l'ensemble $A_{k}$ est fini dans la direction du $k$-ème axe. Je me propose d'établir dans cette note une propriété générale des alephs, qui implique en particulier le théorème de Sierpiński (cas où $a=0$ et $n=2$ ). Cette propriété permet de définir les nombres $s_{n}$ pour $n=0,1,2, \ldots$ sans faire intervenir ni les nombres cardinaux, ni les nombres ordinaux.

Theorème. L'inégalité $\overline{\bar{Z}}<\aleph_{\alpha+n}$ équivaut à l'existence d'un système de $n+1$ ensembles $A_{1}, \ldots, A_{n+1}$ tels que

$1^{0}: Z^{n+1}=A_{1}+\ldots+A_{n+1}$,

$2^{0}$ : pour $k \leqslant n+1$, l'ensemble $A_{k}$ est de puissance $<\boldsymbol{\aleph}_{a}$ dans la direction du k-ème axe.

1) Conférence faite à Praha le 28 mars 1951. Présenté partiellement à la Société Polonaise de Mathématiques, Section de Varsovie, le 9 mars 1951.

2) Voir C. R. Paris 232 (1951), p. 1046, et ce volume, p. 1.
Démonstration. Procédons par induction relativement à $n$. Le théorème étant évident pour $n=0$, admettons qu'il soit vrai pour un entier $n-1 \geqslant 0{ }^{3}$ ).

1) La condition est nécessaire. Posons, par définition, pour tout nombre ordinal $\gamma$,

On a done

$$
Z(\gamma)=\underset{\xi}{E}(\xi<\gamma)
$$

$$
\overline{\overline{Z\left(\omega_{\beta}\right)}}=\aleph_{\beta}, \quad \text { (2) } \quad \overline{\overline{Z(\gamma)}}<\aleph_{\beta} \text { pour } \quad \gamma<\omega_{\beta},
$$

$\omega_{\beta}$ désignant le nombre initial de puissance $\mathbf{s}_{\beta}$.

Pour tout $\gamma<\omega_{\alpha+n-1}$, il existe donc par hypothèse (et d'après (2)) un système de $n$ ensembles $A_{\gamma, 1}, \ldots, A_{\gamma, n}$ tels que:

$$
Z^{n}(\gamma+1)=A_{\gamma, 1}+\ldots+A_{\gamma, n},
$$

(4) pour $r \leqslant n$, l'ensemble $A_{\gamma_{r},}$ est de puissance $<\aleph_{\alpha}$ dans la direction du $r$-ème axe.

En posant $Z=Z\left(\omega_{\alpha+n-1}\right)$, ce qui est évidemment légitime, nous définirons à présent les ensembles $A_{1}, \ldots, A_{n+1}$ de façon à satisfaire aux conditions $1^{0}$ et $2^{0}$. À savoir: nous admettons par définition que l'élément $\left(\xi_{1}, \ldots, \xi_{n+1}\right)$ de $Z^{n+1}$ appartient à $A_{k}$ (où $k \leqslant n+1)$ lorsque l'un des deux eas se présente:

(I) il existe un indice $j>k$ tel que:

(i) $\xi_{j} \geqslant \xi_{m}$ pour $m \leqslant n+1, \ldots$ (i') $\left(\xi_{1}, \ldots, \xi_{j-1}, \xi_{j+1}, \ldots, \xi_{n+1}\right) \in A_{\xi_{j}, k}$,

(II) il existe un $j<k$ tel que:

(ii) $\xi_{j} \geqslant \xi_{m}$ pour $m \leqslant n+1, \quad$ (ii') $\left(\xi_{1}, \ldots, \xi_{j-1}, \xi_{j+1}, \ldots, \xi_{n+1}\right) \in \mathcal{A}_{\xi_{j}, k-1}$.

La condition $1^{0}$ est satisfaite. En effet, étant donné un élément $\left(\xi_{1}, \ldots, \xi_{n+1}\right)$ de $Z^{n+1}$, soit $\xi_{j}$ sa plus grande "coordonnée" (s'il $y$ en a plusieurs, leur choix est arbitraire).

Comme $\left(\xi_{1}, \ldots, \xi_{j-1}, \xi_{j+1}, \ldots, \xi_{n+1}\right) \in Z^{n}\left(\xi_{i}+1\right)$, il existe d'après (3) un indice $r \leqslant n$ tel que

$$
\left(\xi_{1}, \ldots, \xi_{j-1}, \xi_{j+1}, \ldots, \xi_{n+1}\right) \in A_{\xi_{j}, r} .
$$

Si l'on a $j>r$, on est dans le cas' (I) en posant $k=r$, et par conséquent

$$
(5) \quad\left(\xi_{1}, \ldots, \xi_{n+1}\right) \in A_{k} .
$$

3) Pour $n=1$, cf. H. Tietze, Beiträge zur allgemeinen Topologie I, Math. Ann. 88 (1923), p. 306. 
Si l'on a $j \leqslant r$, on posera $k=r+1$. Le cas (II) se trouve alors réalisé, d'où résulte également la formule (5).

Passons à la démonstration de la condition $2^{0}$.

Soit $k$ un entier fixe $\leqslant n+1$. Étant donné un système de $n$ nombres ordinaux $\left(<\omega_{\alpha+n-1}\right): \xi_{1}, \ldots, \xi_{k-1}, \xi_{k+1}, \ldots, \xi_{n+1}$, posons - en considérant ce système comme fixe -

$$
C=E_{\xi_{k}}\left\{\left(\xi_{1}, \ldots, \xi_{k-1}, \xi_{k}, \xi_{k+1}, \ldots, \xi_{n+1}\right) \in A_{k}\right\}
$$

Il s'agit de prouver que $\overline{\bar{C}}<\boldsymbol{s}_{\alpha}$.

Désignons par $B_{j}$ l'ensemble des $\xi_{k}$ tels que le système $\left(\xi_{1}, \ldots, \xi_{n+1}\right)$ satisfait à (i) et (i') ou à (ii) et (ii'), suivant que $j>k$ ou $j<k$. D'après la définition de $A_{k}$, on a donc

$$
C=B_{1}+\ldots+B_{k-1}+B_{k+1}+\ldots+B_{n+1} .
$$

Il reste à prouver que

$$
\overline{\bar{B}}_{j}<\aleph_{\alpha} \text { pour } j=1, \ldots, k-1, k+1, \ldots, n+1 .
$$

Deux cas sont à considérer:

Si $j>k$, c'est-à-dire, si $k$ est l'un des nombres $1, \ldots, j-1$, et la relation $\left(i^{\prime}\right)$ se présente, l'inégalité $(6)$ résulte de $(4)$.

Si $j<k$, c'est-à-dire, si $k$ est l'un des nombres $j+1, \ldots, n+1$, et la relation (ii') se présente, on déduit (6) de (4) en posant

$$
\eta_{1}=\xi_{1}, \ldots, \eta_{j-1}=\xi_{j-1}, \eta_{j}=\xi_{j+1}, \ldots, \eta_{n}=\xi_{n+1} .
$$

2) La condition est suffisante. Supposons, par impossible, que l'ensemble $Z=Z\left(\omega_{\alpha+n}\right)$ admette une décomposition satisfaisant aux conditions $1^{0}$ et $2^{0}$. Posons

$$
S=\sum_{\xi_{n+1}} E\left[\left(\xi_{1}, \ldots, \xi_{n+1}\right) \in A_{n+1}\right],
$$

la sommation étant étendue à tous les éléments $\left(\xi_{1}, \ldots, \xi_{n}\right)$ de l'ensemble $Z^{n}\left(\omega_{\alpha+n-1}\right)$. L'ensemble $A_{n+1}$ étant, d'après $2^{0}$, de puissance $<\aleph_{\alpha}$ dans la direction du $n+1$-ème axe, il vient (c.f. (1)):

$$
\overline{\bar{S}} \leqslant \aleph_{\alpha+n-1}^{n} \cdot \aleph_{\alpha}=\aleph_{\alpha+n-1}<\overline{\bar{Z}} \text {. }
$$

Il en résulte l'existence d'un $\beta \in(Z-S)$. Autrement dit,

$$
Z^{n}\left(\omega_{\alpha+n-1}\right) \times(\beta) \subset Z^{n+1}-A_{n+1} \subset A_{1}+\ldots+A_{n}
$$

d'après $1^{0}$.
Posons pour $k \leqslant n$ :

$$
A_{k}^{*}=\underset{\xi_{1} \ldots \xi_{n}}{E}\left[\left(\xi_{1}, \ldots, \xi_{n}, \beta\right) \in A_{k}\right]
$$

(l'ensemble $A_{k}^{*} \times(\beta)$ est donc l'intersection de $A_{k}$ avee le "plan" $\left.\xi_{n+1}=\beta\right)$.

$$
\begin{aligned}
& \text { Il vient, d'après (7), } \\
& \qquad Z^{n}\left(\omega_{\alpha+n}\right) \subset A_{1}^{*}+\ldots+A_{n}^{*} .
\end{aligned}
$$

Chacun des ensembles $A_{k}^{*}$ (où $k \leqslant n$ ) étant de puissance $<\boldsymbol{N}_{\alpha}$ dans la direction du $k$-ème axe (d'après $\left.2^{0}\right)$ et $Z\left(\omega_{\alpha+n-1}^{*}\right)$ étant de puissance $\aleph_{\alpha+n-1}$, on parvient ainsi à une contradiction avec l'hypothèse que la condition envisagée est suffisante dans le cas de $n-1$.

Corollaire. Pour que l'ensemble $Z$ soit de puissance $\boldsymbol{s}_{n}$ ( $n$ entier $\geqslant 0$ ), il faut et il suffit que l'ensemble $Z^{n+2}$ admette une décomposition en $n+2$ ensembles $A_{1}, \ldots, A_{n+2}$, où $A_{k}$ est fini dans $l a$ direction du k-ème axe et que, cependant, l'ensemble $Z^{n+1}$ n'admette pas de décomposition de ce genre en $n+1$ ensembles. 